\title{
Embryonic Stem Cells Overexpressing Pitx2c Engraft in Infarcted Myocardium and Improve Cardiac Function
}

\author{
AK. Guddati, ${ }^{1 *}$ MD, José Javier Otero, ${ }^{2 *}$ MD, Eric Kessler, ${ }^{3}$ MD, \\ Gary Aistrup, ${ }^{4} \mathrm{PhD}$, J. Andrew Wasserstrom, ${ }^{5} \mathrm{PhD}$, Xiaoqiang Han, ${ }^{6} \mathrm{MD}$, \\ Matthew J. WebBer, ${ }^{7}$ BS, Samuel I. Stupp, ${ }^{7}$ PhD, Jon W. Lomasney, ${ }^{6}$ MD, \\ and John A. KesSLER, ${ }^{1}$ MD.
}

\section{SUMMARY}

This study investigated the effects on cardiomyocyte differentiation of embryonic stem cells by the overexpression of the transcription factor, Pitx $2 \mathrm{c}$, and examined the effects of transplantation of these differentiated cells on cardiac function in a mouse model of myocardial infarction.

Pitx2c overexpressing embryonic stem cells were characterized for cardiac differentiation by immunocytochemistry, RNA analysis, and electrophysiology. Differentiated cells were transplanted by directed injection into the infarcted murine myocardium and functional measurements of blood pressure, contractility, and relaxation were performed. Histochemistry and FISH analysis performed on these mice confirmed the engraftment and cardiac nature of the transplanted cells.

Pitx2c overexpressing embryonic stem cells robustly differentiated into spontaneously contracting cells which acquired cardiac protein markers and exhibited action potentials resembling that of cardiomyocytes. These cells could also be synchronized to an external pacemaker. Significant improvements $(P<0.01)$ in blood pressure $(56 \%)$, contractility $(57 \%)$, and relaxation $(59 \%)$ were observed in infarcted mice with transplants of these differentiated cells but not in mice which were transplanted with control cells. The Pitx2c overexpressing cells secrete paracrine factors which when adsorbed onto a heparinated gel and injected into the infarcted myocardium produce a comparable and significant $(P<0.01)$ functional recovery.

Pitx $2 \mathrm{c}$ overexpression is a valuable method for producing cardiomyocytes from embryonic stem cells, and transplantation of these cardiomyocytes into infracted myocardium restores cardiac function through multiple mechanisms. (Int Heart J 2009; 50: 783-799)

Key words: Pitx2c, Embryonic stem cells, Myocardial infarction, Cardiac differentiation, Cell transplantation

From the ${ }^{1}$ Department of Neurology, Feinberg School of Medicine, Northwestern University Chicago, ${ }^{2}$ Department of Pathology, University of California, San Francisco, ${ }^{3}$ Department of Cardiology, University of Chicago, Departments of ${ }^{4}$ Molecular Pharmacology and Biological Chemistry, ${ }^{5}$ Cardiology, ${ }^{6}$ Pathology, Feinberg School of Medicine and ${ }^{7}$ Department of Biomedical Engineering Northwestem University, Chicago, USA.

* These two authors contributed equally.

Address for correspondence: AK Guddati, MD, Harlem Hospital Center at Columbia University, Department of Internal Medicine, 506 Lenox Avenue, New York, NY 10037, USA.

Received for publication May 29, 2009.

Revised and accepted August 13, 2009. 
TRANSPlanTATION strategies for repairing the damaged heart have employed a number of different cell types including skeletal muscle progenitor cells, fetal derived cardiomyocytes, mesenchymal stem cells, and embryonic stem (ES) cells. ${ }^{1)}$ Although there are problems with each of these cell types, ES cells show particular promise as a source of cells for myocardial repair. ${ }^{2,3)} \mathrm{ES}$ cells can be generated in large quantities, and ES cell-derived cardiomyocytes possess the phenotypes of cardiomyocytes, such as morphology, electromechanical coupling, and the presence of gap junctions. ${ }^{4-6)}$ ES cell differentiation into cardiomyocytes is facilitated by formation of embryoid bodies (EBs), aggregated clusters of cells that form cystic structures with layers of ectodermal, mesodermal, and endodermal lineages. ${ }^{7)}$ However the heterogeneous mixture of cells present after EB formation yields only about 1-3\% cardiomyocytes, and the generation of beating EBs is not reliable for cell transplantation purposes. ${ }^{8)}$ Multiple protocols have been devised to increase the efficiency of cardiomyocyte differentiation and to obtain purified preparations of cardiomyocytes by genetic selection or mechanical dissection of beating clusters of EBs. ${ }^{9)}$ Such ES cell-derived cardiomyocytes can integrate into infarcted myocardium after transplantation and improve ventricular function. ${ }^{10)}$ However the protocols for generating ES cell-derived cardiomyocytes are still cumbersome and relatively inefficient, and this limits the potential for using these cells for transplantation. Thus, the creation of a reliable method for generating large numbers of cardiomyocytes would be an important step towards development of methodologies for using transplanted cells to regenerate damaged myocardium.

Cardiomyocyte differentiation of ES cells can be fostered by manipulating the expression of transcription factors and their signaling cascades. For example, ES cells deficient for RBP/J, a protein involved in the transduction of the notch signaling cascade, show enhanced cardiomyocyte differentiation. ${ }^{11)}$ Also, expression of GATA-4, a $\mathrm{Zn++}$ finger transcription factor and Tbx5, a T-box transcription factor involved in heart development, increases cardiomyocyte differentiation of P19 embryonal carcinoma cells. ${ }^{12,13)}$ Myocyte differentiation of P19 cells is also fostered by treatment with DMSO which leads to an increase in canonical wnt signaling. Conversely, inhibition of endogenous wnt signaling by treatment with frizzled receptor body inhibited DMSO induced myogenesis. ${ }^{14)}$ In this study, we direct ES cells to differentiate into the cardiomyocyte lineage by expression of the bicoid related homeobox gene Pitx $2 c$, a wnt/ $\beta$-catenin target gene. ${ }^{15,16)}$

Pitx2c plays a crucial role in the establishment of left-right asymmetry in the developing heart. ${ }^{17,18)}$ Pitx $2 \mathrm{c}$ expression begins in the left cardiac crescent, a population of cardiac progenitor cells, and in E8 mouse embryos (tubular heart 
stage), Pitx2c expression is restricted to the left side of the tubular heart. By E10.5, expression of Pitx2c is observed in the left inflow tract, the left atrial appendage myocardium, and the left atrioventricular canal myocardium. Loss of Pitx2c function leads to atrial isomerism, double inlet left ventricle, transposition of the great arteries, persistent truncus arteriosus, and abnormal aortic arch remodeling. ${ }^{18,19)}$ Patients suffering from Axenfeld-Rieger syndrome, an autosomal dominant disease resulting from mutations in the homeodomain of Pitx2c, can suffer from defects in cardiac development. ${ }^{20,21)}$ Pitx2c overexpression in skeletal myoblasts promotes cell proliferation and decreases differentiation. ${ }^{22)}$ Because of its role in cardiac development, we investigated the potential of Pitx2c in inducing cardiac differentiation of ES cells to form functioning cardiac myocytes. We find that overexpression of Pitx2c induces differentiation of ES cells into cardiomyocytes that exhibit spontaneous contractions and action potentials similar to normal cardiomyocytes. Further, they engraft in infarcted mouse myocardium leading to functional recovery of contractility and blood pressure. We have also found that hypoxic conditions cause Pitx2c overexpressing cells to secrete paracrine factors which also contribute to functional recovery in a mouse model of myocardial infarction. We conclude that Pitx2c enhances cardiomyocyte differentiation of ES cells, and that transplants of such ESC-derived cardiomyocytes can be used to repair damaged myocardium.

\section{METHODS}

Cell culture and differentiation: The R1 ES cell line was obtained from Dr. Rex Chisholm (Northwestern University). ES cells were cultured without feeder cells on gelatin coated tissue culture flasks in media described previously with LIF (ESGRO, Chemicon). Cells were routinely observed for SSEA-1 and Oct4 staining by immunofluorescence and were never kept in continued cell culture for more than 15 passages. For EB inductions, dissociated ES cells were counted by trypan blue exclusion and seeded at $2-5 \times 10^{5}$ cells $/ \mathrm{mL}$ in $10 \mathrm{~cm}$ petri dishes (Falcon) in induction media to enable them to grow in suspension without attaching to the bottom of the dish. Induction media was identical to the cell culture media except that LIF and $\beta$-mercaptoethanol were not added. Cells were grown in suspension as EBs for a total of 8 days; media was replenished every two days. On day 8 of the induction, EBs were dissociated by incubation with $0.25 \%$ trypsin-EDTA (Invitrogen) for 5 minutes and were plated onto laminin or fibronectin coated glass coverslips (with equivalent results) in induction media. One $10 \mathrm{~cm}$ petri dish was plated into 12 wells of a 24 well plate after trypsinization. Spontaneously contracting cell clusters were observed 2 days afterwards and were observed for up to 4 weeks. 
Cloning and RT-PCR: Pitx2c was cloned from day 4 embryoid bodies by RTPCR. Reverse transcription was performed using the Thermoscript RT-PCR kit (Invitrogen) and PCR (30 cycles) was performed. Primers used for both the cloning and the detection of Pitx2c were as follows: forward primer (BamH1 site), 5'-ACG GAT CCA TGA ACT GCA TGA AAG GCC CGC TG-3'; reverse primer (XbaI site), 5'-TTT CTA GAT CAC ACC GGC CGG TCG ACT GC-3'. Template for cloning Pitx2c cDNA was RA treated EBs. All PCR cloning was performed using the Thermoscript PCR, high fidelity kit so as to minimize the number of random mutations.

Quantification: Quantification was conducted by induction of 5 million cells with Pitx2c and an equivalent number of ES cells with empty vector. EBs were cultured for 8 days in suspension and plated on fibronectin on the $8^{\text {th }}$ day. The plated EBs were cultured for two days and fixed with $4 \%$ paraformaldehyde for immunostaining. EBs with more than 50\% cardiac troponin T (Santa Cruz Biotech, CA) positive staining were counted. The same protocol was applied for two different Pitx2c expressing ES cell lines to control for the site of integration of the transgene. Three hundred EBs were counted for each clone and statistical analysis for significant difference was determined by the Bonferroni and Dunn tests.

Real time quantitative PCR: Total cellular RNA was extracted using Trizol (Invitrogen, Carlsbad, CA) reagent according to the protocol provided by the company. Trace amounts of genomic DNA were removed using a Turbo DNAfree kit (Ambion). 18S was used as an internal control and all reactions were performed on an ABI 7700 Prism system. Fold changes of mRNA levels were calculated using the delta delta $\mathrm{Ct}$ method after verifying the linear range of amplification. All time points were run in duplicate and 3 such independent studies were performed. ES cells transfected with empty vector were used as controls for comparison of fold changes of cardiac specific mRNA levels. PCR products were verified by melting temperature dissociation curves and by DNA sequencing.

Generation of stable cell lines: The effects of Pitx2c on cardiac lineage commitment by ES cells were examined by transfecting the cells with full length Pitx2c. Since ES cells undergo several rounds of mitosis during the process of differentiation in vitro, stably transfected cell lines were established to avoid possible effects due to dilution of transiently transfected cells and/or preferential proliferation of untransfected cells. The genes of interest were placed in the pcDNA3.1 expression plasmid (Invitrogen) under the control of a CMV promoter. Constitutively active promoters are widely used to study in vitro differentiation of ES cells. Cells were transfected by electroporation. Selection media containing 250 $\mu \mathrm{g} / \mathrm{mL}$ geneticin (Life Technologies) was added 2-3 days postelectroporation. 
Colonies of geneticin resistant ES cells were picked and expanded as individual colonies in 96 well plates. These colonies were inspected daily for differentiation and selection medium was changed. On day 8 of postplating, the colonies were dissociated with $0.5 \%$ trypsin and plated as duplicates. One set of plates were used for genomic analysis for plasmid insertion and the other set of plates were frozen at $-80^{\circ} \mathrm{C}$. Genomic DNA was extracted by proteinase K digestion and analyzed for insertion of plasmid DNA by PCR. PCR positive clones were identified and extracted from the frozen duplicate plates. These colonies were expanded in selection media while differentiation experiments were performed in media without geneticin. Pitx $2 \mathrm{c}$ overexpression was verified by RT-PCR. Each stable cell line was generated from each colony. Stable cell lines generated were routinely tested by PCR of genomic DNA to ensure continued integration of the construct (data not shown). None of the cell lines were observed to alter expression of SSEA-1 and Oct4 or alter undifferentiated morphology (data not shown). All experiments were repeated in independently derived clones to control for possible positional effects.

Intracellular $\mathrm{Ca}^{2+}$ imaging: Intracellular $\left[\mathrm{Ca}^{2+}\right]$ was measured as the fluorescence intensity of the $\mathrm{Ca}^{2+}$ indicator Fluo-4AM. Fluorescence was measured on the stage of a laser scanning confocal microscope (Zeiss LSM 510). Beating cultures were incubated at $37^{\circ} \mathrm{C}$ for 30 minutes in DMEM containing $40 \mu \mathrm{M}$ Fluo-4AM. The coverslip was then placed in an experimental chamber on the microscope stage and perfused with DMEM $\left(32^{\circ} \mathrm{C}\right)$. Spontaneously beating EBs were exposed to light from a $25 \mathrm{~mW}$ argon laser $(488 \mathrm{~nm})$ and emitted light (> $505 \mathrm{~nm}$ ) was collected. A scan line was drawn across an individual EB and fluorescence light intensity was measured from the line at a scan rate of $2 \mathrm{msec}$ in order to build a 2-D image (time versus distance) of fluorescence intensity across the various cells in the EB of interest. Fluorescence intensity is presented as a ratio compared to intensity at rest immediately before activation of $\mathrm{Ca}^{2+}$ transients (F/F0). Platinum field stimulating electrodes were placed $1 \mathrm{~mm}$ from either side of the EB in order to apply square pulse stimulation $(2 \mathrm{~Hz}$, pulse duration $5 \mathrm{msec}, 1.2 \mathrm{x}$ threshold) across the EB. Both spontaneous and stimulated fluorescence transients were recorded from each EB whenever possible.

Action potential measurements: Beating EBs were placed in an experimental chamber on the stage of an inverted microscope (Nikon Diaphot). The chamber was constantly perfused with bubbled $\left(95 \% \mathrm{O}_{2}: 5 \% \mathrm{CO}_{2}\right)$ DMEM maintained at $36^{\circ} \mathrm{C}$. Recordings were made using high resistance microelectrodes (25-50 megohms) pulled from borosilicate capillary tubing (1B100F, World Precision Instruments) on a horizontal puller (P-87, Sutter Instruments). Microelectrodes were filled with $1 \mathrm{M} \mathrm{KCl}$ and fitted into a holder attached to the headstage of an Axoclamp-2 amplifier (Axon Instruments). Data acquisition and analysis and 
stimulation protocols were performed using pClamp6 software using a TL-125 DMA interface (Axon Instruments). Cell stimulation $(2 \mathrm{~Hz})$ was accomplished by delivering $2 \mathrm{msec}$ pulses (1.2x threshold) through the pipette in bridge mode. Immunocytochemistry: Cells plated on cover slips (Carolina Science \& Math) were fixed in $4 \%$ paraformaldehyde and permeabilized with $0.2 \%$ Triton X-100. Primary antibodies used were the following: anti-cardiac specific troponin-T (Santa Cruz), anti-Nkx 2.5 (Santa Cruz), anti-GATA4 (Santa Cruz), anti-Oct4 (BD Pharmingen), and anti-SSEA-1 (Developmental Studies Hybridoma Bank). Appropriate secondary antibodies were purchased from Southern Biotechnology Associates. Cover slips were mounted onto glass slides (Fisher) with Antifade Kit (Molecular Probes).

Cell transplantation: Pitx2c overexpressing ES cells and R1 ES cells were differentiated into embryoid bodies by withdrawal of LIF in the medium. Both cell types were differentiated using exactly the same protocol. Eight day old embryoid bodies were trypsinized with $0.25 \%$ Trypsin-EDTA (Gibco) and washed with PBS and approximately $1 \times 10^{6}$ cells were directly injected into the wall of the infarcted left ventricle. The study group consisted of 15 mice divided into 4 groups ( $n=3$ for 3 groups and $n=6$ for healthy controls): Infarcted mice receiving differentiated Pitx $2 \mathrm{c}$ expressing cells, infarcted mice receiving differentiated untransfected R1 ES cells, infarcted mice, and healthy mice. Myocardial infarction was induced in $129 \mathrm{SvJ}$ female mice anaesthetized by ketamine after which the chest was opened and the left anterior descending coronary artery was transiently ligated 2-3 mm from the origin of the LAD for 30 minutes and the circulation was restored thereafter. Infarcted mice were monitored for one week and the cell transplantations were performed on the eighth day of postinfarction by direct injection into the myocardial scar tissue in the left ventricle. Mice were evaluated for functional improvement after one month of cell transplantation. Blood pressure, contractility (dP/dt), and relaxation (-dP/dt) were measured with a SPR-839 microcatheter using a Millar Pressure-Volume system. Pressurevolume curves were analyzed on PVAN software provided by Millar. Mice were sacrificed after functional studies and infarct size was inspected after fixing the heart. Triphenyltetrazolium staining of the infarcted hearts revealed approximately $44 \%$ ischemic area (IA) in the area at risk (AAR) (IA/AAR $=44.6 \pm 4.6$ ) and approximately $43 \%$ area at risk in the left ventricle (AAR/LV $=43 \pm 3.2 \%$ ). FISH analysis and immunoflourescence measurements were performed to verify the presence of differentiated transplanted Pitx2 overexpressing cells with cardiac phenotype. All procedures were approved and were conducted according to Institutional guidelines.

Conditioned media loading of a peptide amphiphile scaffold: Heparin Binding Peptide Amphiphile (HBPA: $\mathrm{C}_{16} \mathrm{H}_{31} \mathrm{O}$-AAAAGGGLRKKLGKA- $\mathrm{NH}_{2}$ ) was syn- 
thesized as previously described ${ }^{23)}$ and purified using reversed phase high performance liquid chromatography. A solution of HBPA $(3 \% \mathrm{w} / \mathrm{v})$ was triggered to self-assemble by mixing with an equal volume of a solution of heparin sodium salt derived from porcine intestinal mucosa (Sigma-Aldrich, $2 \% \mathrm{w} / \mathrm{v}$ ). The resulting gel was allowed to cure for 30 minutes at room temperature. Hypoxicconditioned Pitx2 media was prepared by culturing Pitx2 cells at 5\% $\mathrm{O}_{2}$ for 24 hours, at which point the media was removed, centrifuged, and stored at $-80^{\circ} \mathrm{C}$ for future use. The cured HBPA gel was soaked in $1 \mathrm{~mL}$ of this media at $4^{\circ} \mathrm{C}$ for 24 hours. The gels were used as a treatment in a mouse myocardial infarction model (CD1 mice from Charles River). Following tracheal intubation, the heart was exposed and the left anterior descending artery was ligated for 30 minutes. After this time, $15 \mu \mathrm{L}$ of gel was injected, and the tissue was reperfused. Animals were phenotyped ( $n=5$ per group, $n=10$ for healthy controls) for functional performance at 30 days using a pressure-volume catheter inserted into the left ventricle through the right carotid artery. Control measurements were done on healthy animals, animals receiving an infarction without treatment, and animals receiving HBPA gels soaked in unconditioned media (serum-free DMEM). FISH analysis: Four micron thick sections of the fixed cardiac tissue were stained for the Y chromosome using a FITC labeled probe (Open Biosystems) using the protocol provided by the company. These sections were immediately adjacent to those used for immunostaining for Troponin- $\mathrm{T}$ (Figure $4 \mathrm{~F}$ and $\mathrm{E}$ are adjacent sections at different magnifications).

Statistical analysis: Statistical tests were conducted with post-hoc ANOVA tests. A $P$ value less than 0.05 was considered to be statistically different and all values are reported as means with positive and negative standard deviations, except electrophysiological measurements which are reported as the mean \pm standard error of the mean.

\section{RESULTS}

Pitx2c induces robust cardiomyocyte differentiation: ES cells transfected with Pitx2c were grown as separate stable cell lines and Pitx2c expression was found to be enriched approximately 750 fold $(748.3 \pm 80$ ) by RT-PCR (Figure $1 \mathrm{G}$ ). These cell lines were induced to differentiate by seeding them at $2-5 \times 10^{5}$ cells/ $\mathrm{mL}$ in induction media to form EBs which were grown in suspension for 8 days. Cells transfected with an empty vector were used as controls. On the $8^{\text {th }}$ day of the induction, the cells were dissociated and plated onto either laminin-1 or fibronectin coated glass coverslips in induction media. Maintenance in induction media promoted cardiomyocyte differentiation. Spontaneously contracting cell clusters were observed 2 days after EB dissociation in the Pitx2c transfected cell 
line but only rarely in the control cell line, and clusters continued to beat spontaneously for over 30 days. The generation of beating foci in the culture dishes was not homogeneous, and we routinely observed that distinct foci began beating at different times. This suggests that cardiomyocyte differentiation did not occur at the same rate in all of the EBs in spite of the homogeneity in their size. Furthermore, beating cells originated in the center of large clusters, suggesting that Pitx2c did not induce cardiomyocytes alone and that other factors such as cell-cell contact may be important in cardiomyocyte differentiation of ES cells in vitro. Cells were fixed and stained 5-7 days post EB dissociation. Cardiomyocyte differentiation was confirmed by immunostaining the cells for cardiac specific troponin-T and for the transcription factors GATA-4 and Nkx2.5, proteins that are all expressed in cardiomyocytes (Figure 1). The extent of differentiation induced by Pitx $2 \mathrm{c}$ was measured by quantifying the number of cardiac troponin positive EBs of different clones. Approximately 30\% (29.33 $\pm 8.4 \%)$ of the Pitx2c overexpressing EBs were found to contain more than $50 \%$ of cardiac troponin $\mathrm{T}$ positive cells compared to less than $8 \%$ of the control group of EBs consisting of empty vector transfected ES cells.

The time course of cardiac specific differentiation was studied by performing a time series analysis of various cardiac specific mRNA levels of Pitx 2c transfected cells and control cells with real time quantitative PCR (Figure 2). Transcription factors known to be involved in cardiogenesis, Nkx2.5, Mef2c, Tbx5, and Mef2c, were significantly upregulated in the Pitx2c transfected cells compared to empty vector-transfected controls (Figure 2A). Transcripts for structural proteins like MLC2a, MLC2v, and Cardiac Troponin I were also significantly elevated during a differentiation protocol of 10 days. Similarly, RTPCR of control and Pitx2c transfected cells showed a clear decrease in Oct 3/4 by day 3 of differentiation and a complete loss by day 8 which indicates that most of the cells are differentiated (Figure 2B). Connexin 43 also increased significantly, providing evidence of the potential for formation of gap junctions. The alpha and beta isoforms of myosin heavy chain were also upregulated along with cardiac actin which points towards the production of proteins necessary for the contractile apparatus.

Pitx2c induces the formation of spontaneously contracting cells that exhibit electrophysiological characteristics of cardiomyocytes: The functional properties of these cultured cardiomyocytes were assayed by fluorescent calcium imaging. Figure 3 shows typical recordings of fluorescence intensity across a Pitx2c overexpressing EB using fluo-4AM. Figure 3A shows the location of the line from which cells were sampled repetitively to build the linescan images in subsequent panels. Figure 3B demonstrates that fluo-4AM-loaded cells within this EB generate spontaneous and coordinated increases in cytoplasmic $\left[\mathrm{Ca}^{2+}\right]$ (fluo- 

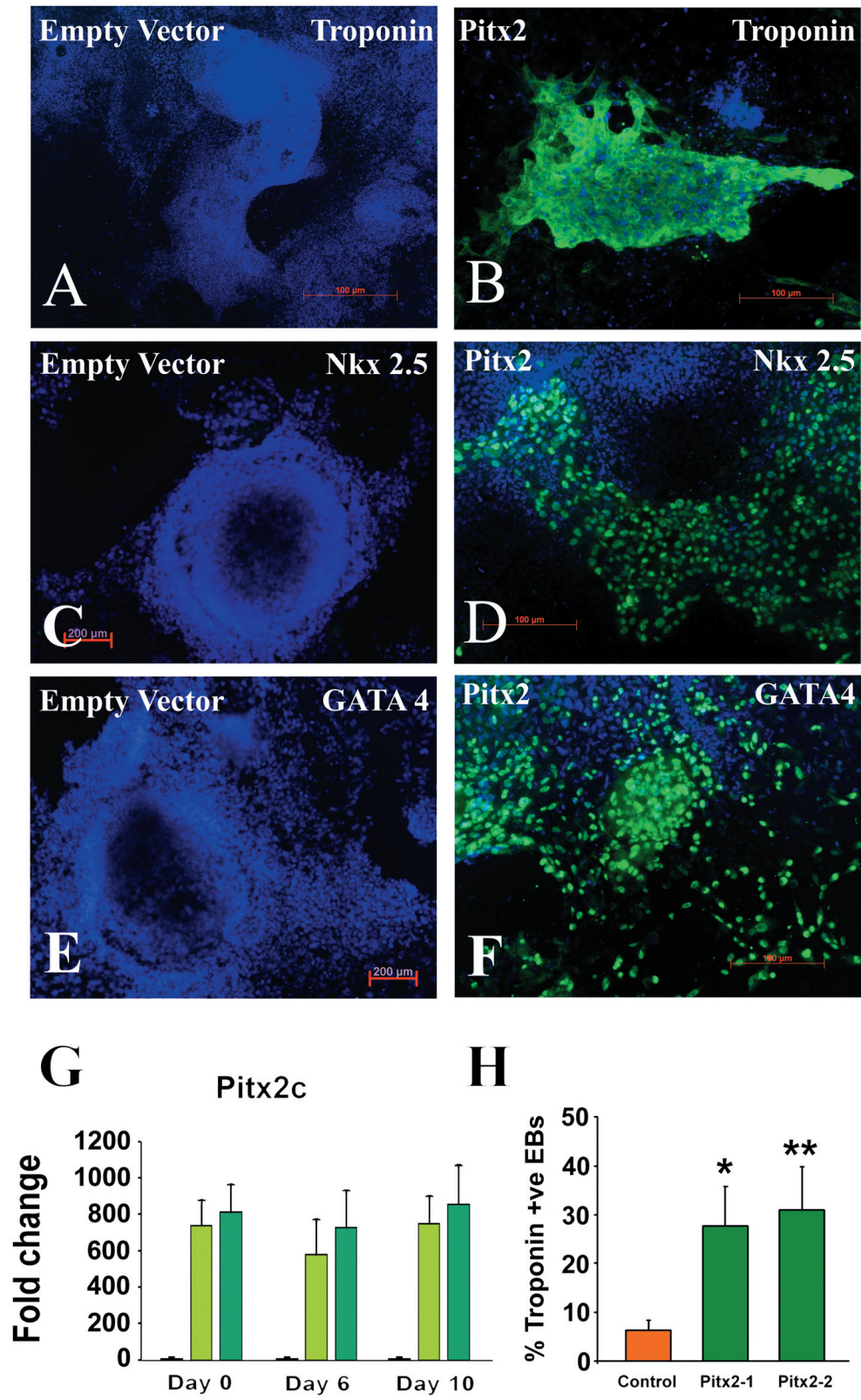

Figure 1. Expression of Pitx2c induces cardiomyocyte differentiation in ES cells. Empty vector transfected cells (A) and Pitx2c transfected cells (B) were fixed and stained for cardiac troponin-T (green) and counterstained with Hoechst to visualize nuclei. Virtually every partially dissociated EB contained clusters of cardiac Troponin-T positive cells. Cells in the Pitx2c transfected cells were positive for the transcription factors GATA-4 (D) and Nkx2.5 (F) when compared to control empty vector transfected cells ( $\mathbf{C}$ and $\mathbf{E})$. Pitx2c expression was elevated in two different Pitx2c transfected cell lines (light green and dark green) when measured by RT-PCR and compared to control cell lines (orange) (G). Quantification of cardiac Troponin T positive EBs in different clones of Pitx 2 transfected cells (dark green) shows a significant (* and $* * P<0.05$ compared to control group by ANOVA) increase compared to control empty vector transfected cells (orange) $(\mathbf{H})$. 
A
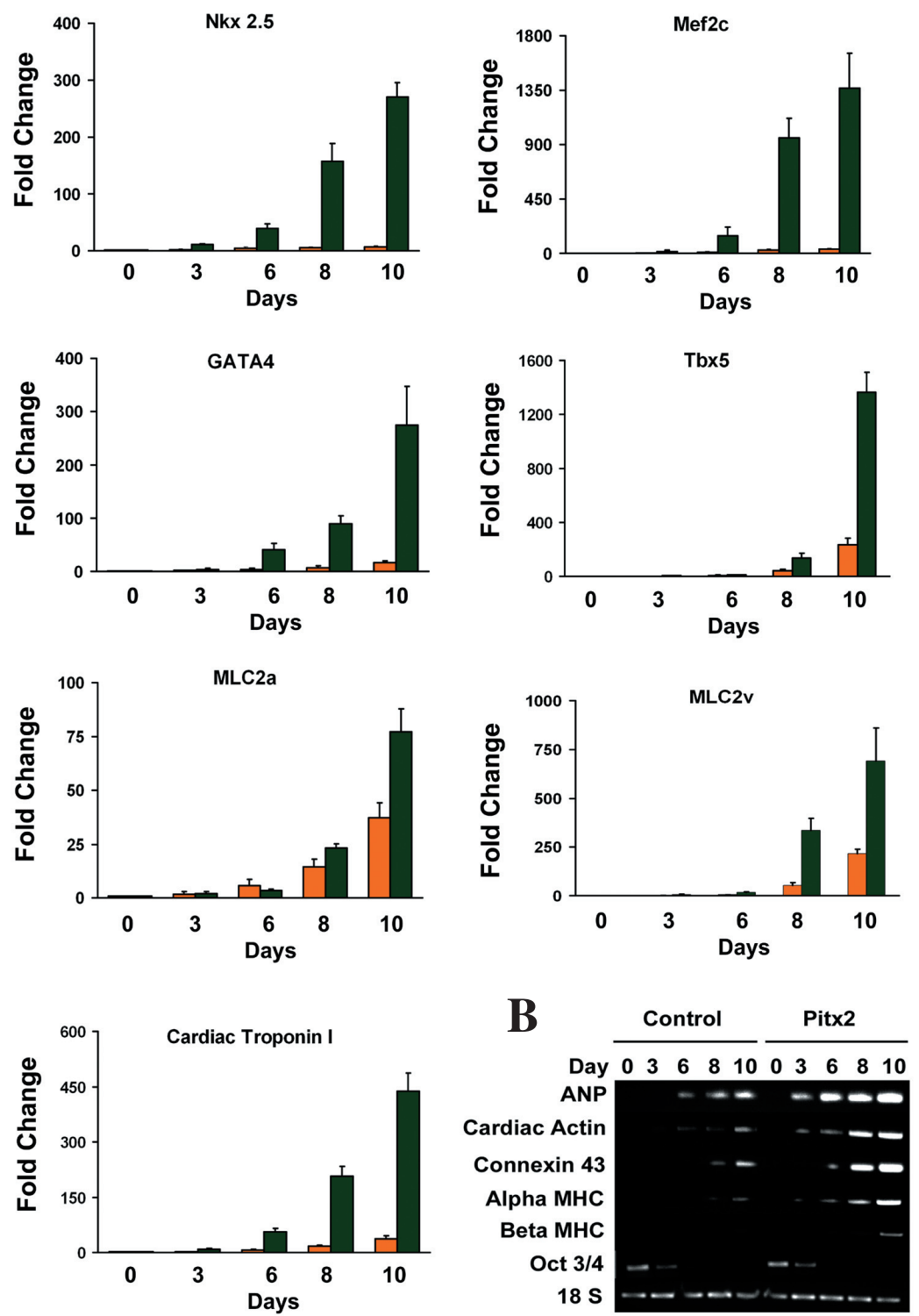

Figure 2. Cardiac specific transcripts are upregulated in Pitx 2c transfected cells. Real time quantitative PCR of various transcripts in control cells with empty vector (orange) and Pitx2c transfected cells (green). It shows an increase in the expression of the mRNA of transcription factors like Nkx2.5, Mef2c, GATA4, and Tbx5. mRNA of structural proteins like MLC2a, MLC2v and Cardiac Troponin I are also upregulated (A). RT-PCR of various cardiac specific transcripts shows a similar trend in the Pitx 2 transfected cells compared to control cells. Oct $3 / 4$ transcripts decrease with differentiation $(\mathbf{B})$. 
rescence intensity). $\mathrm{Ca}^{2+}$ increased abruptly reaching a maximum within a few milliseconds before declining, and the cycle of $\mathrm{Ca}^{2+}$ transients was repeated at a frequency of about $1.25 / \mathrm{sec}$. During field stimulation $(2 \mathrm{~Hz})$, the cells were able to follow the external stimulus (Figure 3C) indicating that excitation of these cells could be evoked by external activation of sarcolemmal ion channels. These $\mathrm{Ca}^{2+}$ transients from both spontaneous and stimulated Pitx2c-ES cell-derived cardiomyocytes resemble those found in cardiomyocytes derived from wild type ES cells.

Figure 3D shows a typical recording of an action potential from an EB after 10 days of beating in culture. In this cell, the maximum diastolic potential

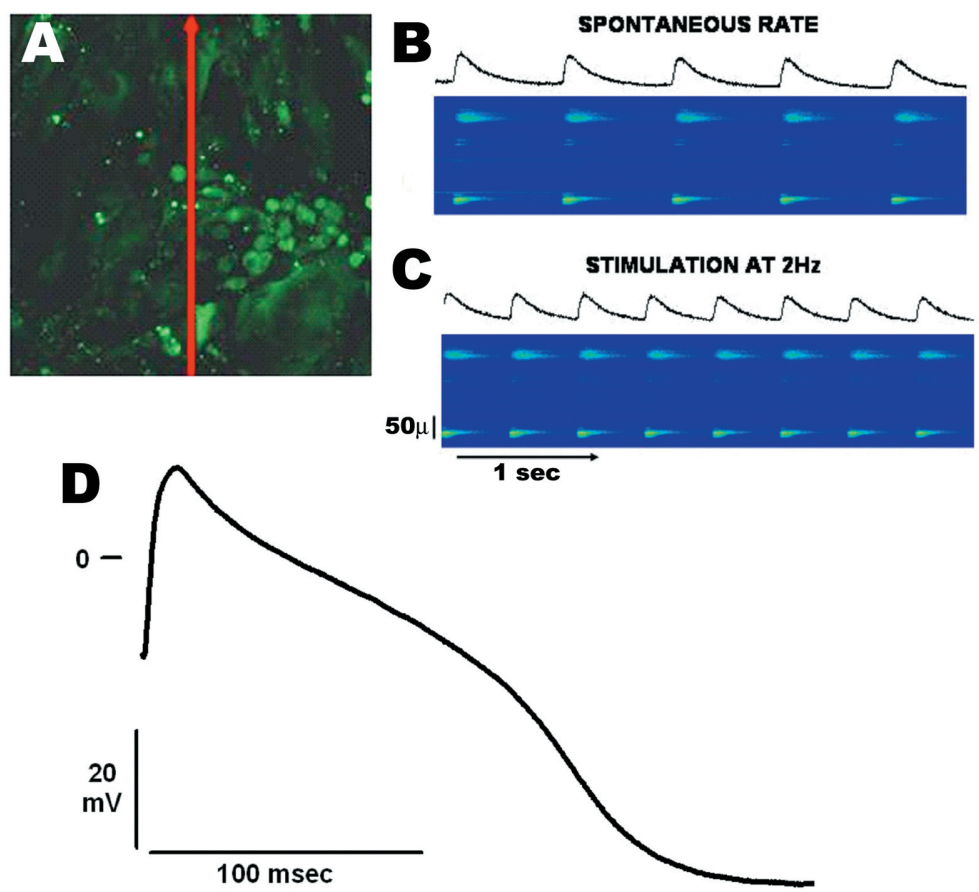

Figure 3. $\mathrm{Ca}^{2+}$ transients and ventricular-like action potentials. A shows a 2-D image of Pitx2c expressing cells in an 8 day EB after loading with fluo-4AM. The red line shows the site of repetitive scanning used to record linescan images in subsequent panels. The scale of the image is 340 micron square. B shows a linescan image recorded by repetitive scanning at site indicated by the red line in $\mathbf{A}$ at a rate of $2 \mathrm{msec} / \mathrm{line}$. Spontaneous $\mathrm{Ca}^{2+}$ transients were activated simultaneously at a number of cells throughout the EB. The integrated profile across the entire image is shown above. When field stimulation $(2 \mathrm{~Hz})$ was initiated $(\mathbf{C}), \mathrm{Ca}^{2+}$ transients were activated in response to each stimulus, indicating the ability of these same cells within the EB to react to external stimulation. D shows an action potential in a cell from an EB induced by Pitx2. Action potentials were recorded by delivery of a current pulse through the microelectrode for $2 \mathrm{msec}$ at $1.2 \mathrm{x}$ threshold at $2 \mathrm{~Hz}$. 
was $-57 \mathrm{mV}$ and overshoot was $16 \mathrm{mV}$, with action potential durations at $50 \%$ and 90\% (APD50 and APD90) of repolarization of 121 and $170 \mathrm{msec}$, respectively. The action potential resembles that of neonatal rather than adult ventricular myocytes, showing a rapid phase of early depolarization (phase 0), an early phase of partial repolarization (phase 1), and a relatively long plateau phase terminated by rapid repolarization. Overall, maximum diastolic potential was -56.0 $\pm 3.6 \mathrm{mV}$, overshoot was $9.4 \pm 2.7 \mathrm{mV}$, amplitude was $65.4 \pm 4.2 \mathrm{mV}$, APD50 was $120.4 \pm 16.5 \mathrm{msec}$, and APD90 was $181.0 \pm 18.9 \mathrm{msec}($ mean $\pm \mathrm{SEM}, n=$ $7)$. No action potentials typical of atrial and nodal cardiomyocytes were detected in any of the recordings, which is consistent with our observation that each EB usually has only one predominant type of cardiomyocytes. These findings indicate that Pitx2c expression is capable of inducing ventricular-like cellular electrophysiological properties in differentiating mouse ES cells.

Pitx2c overexpressing cells contribute to the functional recovery of infarcted murine hearts by multiple mechanisms: Myocardial infarction was induced in female syngeneic $(129 \mathrm{SvJ})$ mice by left anterior descending artery ligation. One week later Pitx2c overexpressing ES cells that had been differentiated for 8 days as embryoid bodies were injected directly into the infarcted ventricular wall of some animals. Others received injections of similarly differentiated wild type R1 ES cells, and others received no injection (myocardial infarction only). Mice receiving Pitx2c overexpressing cells had a significant 57\% improvement in contractility, 59\% improvement in relaxation, and 56\% improvement in blood pressure as compared to the uninjected (myocardial infarction only) group (Figure 4A-C) when observed one month after cell transplantation. By contrast, injection of wild type ES cells produced insignificant changes in any of these measures of cardiac function.

Histological examination of the hearts injected with Pitx2c overexpressing cells demonstrated the presence of transplanted cells in the scar region (Figure 4D) that were not present either in the hearts injected with differentiated wild type ES cells or in hearts that received no cell injections. Some of the transplanted cells located in the middle of the scar tissue had a positive immunostain for cardiac troponin $\mathrm{T}$ (Figure 4E). The presence of transplanted cells in the infarcted tissue was verified by FISH analysis for Y chromosome positive Pitx2c overexpressing cells in the XX genetic background of host myocardium (Figure 4F-G). In addition, we also observed transplanted cells in the scar tissue which formed rosette-like structures which had not differentiated into cardiomyocytes. All mice involved in this experiment were sacrificed after one month; however it is possible that this beneficial effect may not be sustained in the long term. ${ }^{23)}$ It is unlikely that the cells which stained for troponin $\mathrm{T}$ had electromechanically integrated with the host myocardium. To investigate the other possible mecha- 
nisms that might contribute to functional recovery, Pitx $2 \mathrm{c}$ overexpressing cells were subjected to hypoxic conditions for 24 hours in a serum free medium and the supernatant was incubated with a biocompatible gel. Subsequently the gel was injected into the infarcted myocardium (schematic shown in Figure 5A). The gel used was a self assembling amphophile which contained heparin. ${ }^{23)}$ Control experiments involved the usage of unconditioned medium (without serum) with the gel. Functional measurements of contractility and blood pressure taken in these mice after one month of gel injection showed a significant improvement when compared to control mice (Figure 5B). This indicates that

A

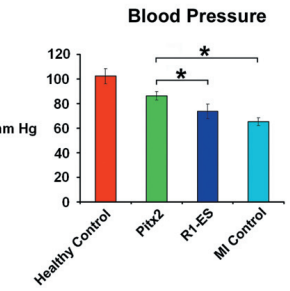

B

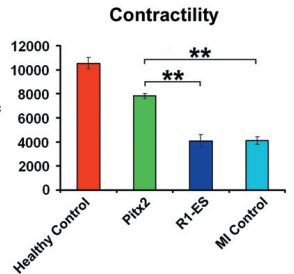

C

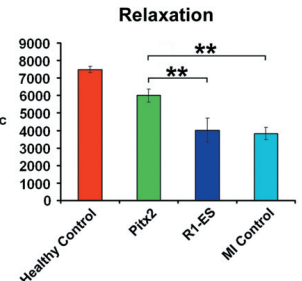

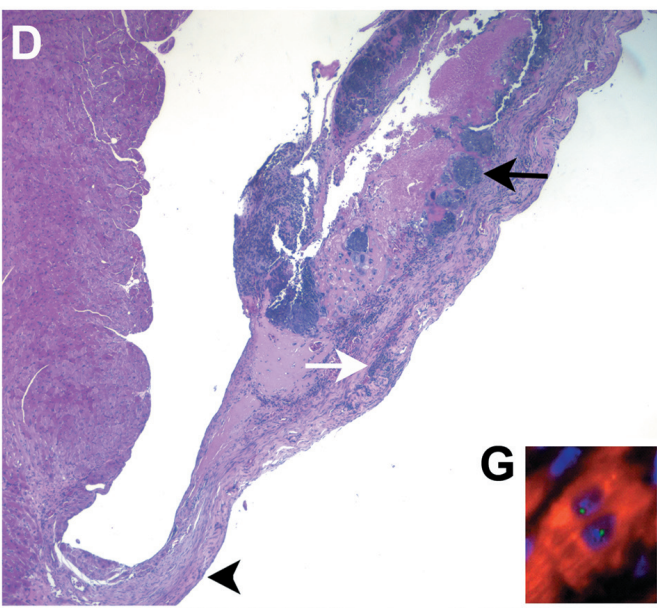
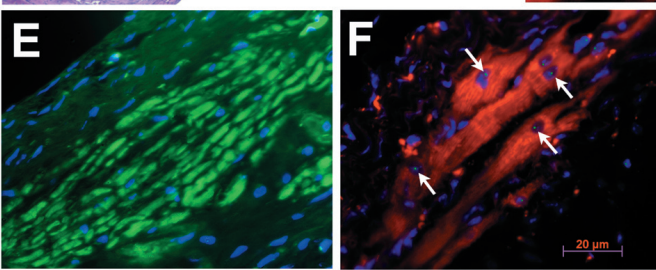

Figure 4. Pitx2c overexpressing ES cells contribute to the functional recovery of infarcted mice. ES cells overexpressing Pitx $2 \mathrm{c}$ were differentiated into embryoid bodies and transplanted into infarcted mice. Functional measurements were done to assess blood pressure, contractility and relaxation $(\mathbf{A}, \mathbf{B}, \mathbf{C})$ one month after cell transplantation $(* P<0.02$ for blood pressure and $* * P<$ 0.001 for contractility and relaxation compared to all other groups by ANOVA, R1-ES cell group and MI control group do not significantly differ from each other). (D) The relatively acellular nature of the infarct is evident at the junction of the infarct (black arrowhead) in an H\&E stain, and the transplanted cells can be seen as in laminar arrangement (white arrow) or as rosettes (black arrow). (E) The same heart was stained for cardiac differentiation of transplanted cells with cardiac troponin $\mathrm{T}$ in the scar $(20 \times)$. (F) The presence of transplanted cells was confirmed by FISH analysis of the Y chromosome with an FITC conjugated probe (white arrows) and cardiac differentiation by cardiac troponin T immunoflourescence (red) (40x). (G) A magnification from $\mathbf{F}$ which shows clear localization of the Y chromosome probe (green) in the nucleus (blue). 
A

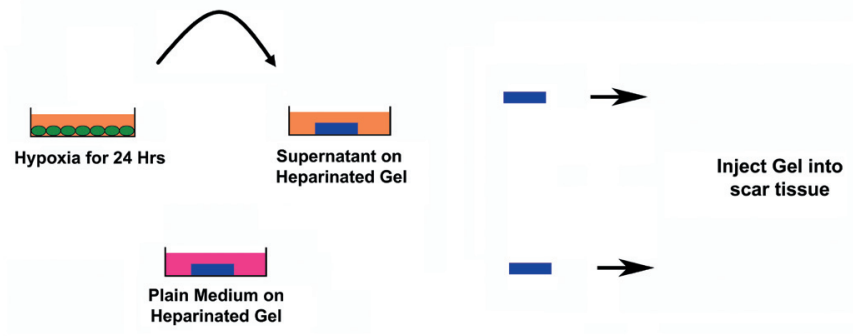

B
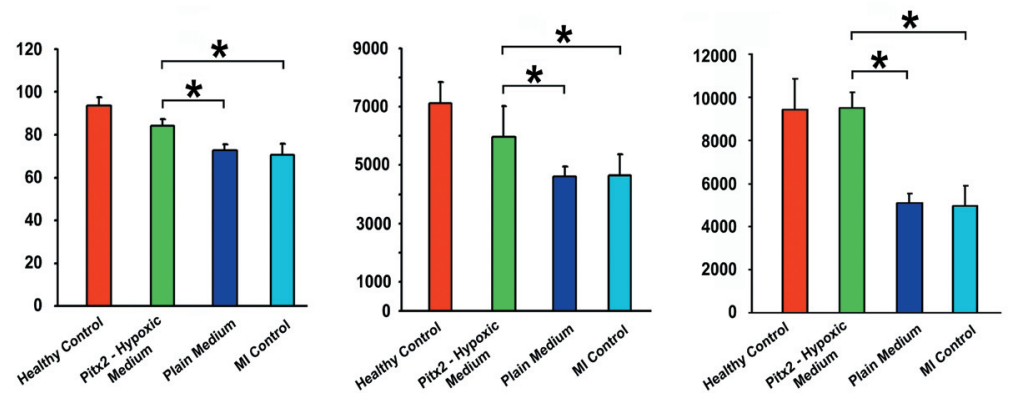

Figure 5. Secreted factors for hypoxic Pitx2c overexpressing cells also contribute to the functional recovery of infarcted mice. (A) Schematic depicting the transfer of the supernatant of hypoxia subjected Pitx2c overexpressing cells onto heparinated gel. (B) Functional measurements were done to assess blood pressure, contractility, and relaxation one month after gel injection (* $P<0.005$ compared to all other groups by ANOVA, plain medium group and MI control group do not significantly differ from each other).

functional recovery due to Pitx2c-ES cell transplantation into the infarcted myocardium may in part reflect paracrine factors secreted by these cells.

\section{Discussion}

A number of techniques have been described for enhancing the differentiation of ES cells into cardiomyocytes, ${ }^{24-27)}$ but the low efficiency and reliability of these techniques has limited their utility for myocardial regeneration. In this study we have found that we can reliably and reproducibly induce cardiomyocyte differentiation of ES cells by overexpressing Pitx2c, a bicoid related homeobox transcription factor involved in the generation of left-right asymmetry of the developing heart and the pulmonary myocardium. ${ }^{28)}$ Pitx $2 \mathrm{c}$ expression enhanced the expression of GATA-4 and Nkx2.5, both of which have been im- 
plicated in the development of cardiomyocytes. ${ }^{29-33)}$ However, overexpression of GATA-4 or Nkx2.5 alone in murine ES cells is insufficient to induce cardiomyocyte differentiation, suggesting that Pitx2c acts through other pathways as well.

Several cell types such as hematopoietic stem cells, ${ }^{34)}$ fibroblasts and primary myocardial cell cultures ${ }^{35)}$ have been used for transplantation to improve recovery of cardiac function in infarcted mice. However, the benefits of such interventions seem to rely more on neoangiogenesis and hence increased perfusion to the damaged ventricular wall rather than direct substitution of infarcted tissue with transplanted cardiomyocytes. In this study, we demonstrate that ES cells with a preferential cardiac differentiation program engraft and differentiate into cardiomyocytes in vivo. We did not observe the formation of teratomas, neoangiogenesis, or evidence of electromechanical coupling of the transplanted cells with the host myocardium. Some of the transplanted cells were observed to be in a laminar arrangement in isolated places in the middle of the scar. Based on measurements of contractility, we believe that the transplanted cells might have actively participated in normal contractions, but some of the beneficial effects might be explained by altered elastic properties of the infarcted tissue after engraftment. Notably, not all of the engrafted cells appeared to adopt the cardiomyocyte phenotype, suggesting that both mechanisms could be responsible for the beneficial effects. We observed that transplanted cells remained engrafted in the myocardium even after one month of transplantation in contradistinction to the common observation that most transplanted cells are lost within the first few days after transplantation. It is possible that some of the improvement in cardiac function was secondary to unknown beneficial paracrine factors arising from Pitx $2 \mathrm{c}$ activation that may also contribute to the continued survival of the transplanted cells, remodeling of the scar tissue, and improved contractility of the residual myocardium which synergistically help in the recovery of the infarcted mice. Similar studies have utilized the direct injection of hypoxic medium from mesenchymal stem cells and have shown functional recovery in rodent models of myocardial infarction. ${ }^{36-38)}$ We tested this possibility of the involvement of paracrine factors by subjecting Pitx2c overexpressing cells to hypoxia and soaking the paracrine factors onto a gel. The injection of this gel in a mouse model of myocardial infarction caused significant functional recovery as compared to mice which received a gel soaked with plain medium.

In summary, overexpression of Pitx2c in ES cells reliably results in differentiation of a high proportion of the cells into cardiomyocytes. Transplantation of these cells into experimentally infarcted myocardium results in engraftment and in significant functional improvement through multiple mechanisms. These findings offer a reliable technique for potentially generating large numbers of cardiomyocytes for regenerative therapies. 


\section{REFERENCES}

1. Murry CE, Field LJ, Menasché P. Cell-based cardiac repair: reflections at the 10-year point. Circulation 2005; 112: 3174-83.

2. Laflamme MA, Gold J, Xu C, et al. Formation of human myocardium in the rat heart from human embryonic stem cells. Am J Pathol 2005; 167: 663-71.

3. Kehat I, Khimovich L, Caspi O, et al. Electromechanical integration of cardiomyocytes derived from human embryonic stem cells. Nat Biotechnol 2004; 22: 1282-9.

4. Hescheler J, Fleischmann BK, Lentini S, et al. Embryonic stem cells: a model to study structural and functional properties in cardiomyogenesis. Cardiovasc Res 1997; 36: 149-62. (Review)

5. Sachinidis A, Fleischmann BK, Kolossov E, Wartenberg M, Sauer H, Hescheler J. Cardiac specific differentiation of mouse embryonic stem cells. Cardiovasc Res 2003; 58: 278-91. (Review)

6. He JQ, Ma Y, Lee Y, Thomson JA, Kamp TJ. Human embryonic stem cells develop into multiple types of cardiac myocytes: action potential characterization. Circ Res 2003; 93: 32-9.

7. Leahy A, Xiong JW, Kuhnert F, Stuhlmann H. Use of developmental marker genes to define temporal and spatial patterns of differentiation during embryoid body formation. J Exp Zool 1999; 284: 67-81.

8. Murry CE, Reinecke H, Pabon LM. Regeneration gaps: observations on stem cells and cardiac repair. J Am Coll Cardiol 2006; 47: 1777-85. (Review)

9. Klug MG, Soonpaa MH, Koh GY, Field LJ. Genetically selected cardiomyocytes from differentiating embronic stem cells form stable intracardiac grafts. J Clin Invest 1996; 98: 216-24.

10. Min JY, Yang Y, Converso KL, et al. Transplantation of embryonic stem cells improves cardiac function in postinfarcted rats. J Appl Physiol 2002; 92: 288-96.

11. Schroeder T, Fraser ST, Ogawa M, et al. Recombination signal sequence-binding protein Jkappa alters mesodermal cell fate decisions by suppressing cardiomyogenesis. Proc Natl Acad Sci U S A 2003; 100: 4018-23.

12. Grépin C, Nemer G, Nemer M. Enhanced cardiogenesis in embryonic stem cells overexpressing the GATA-4 transcription factor. Development 1997; 124: 2387-95.

13. Fijnvandraat AC, Lekanne Deprez RH, Christoffels VM, Ruijter JM, Moorman AF. TBX5 overexpression stimulates differentiation of chamber myocardium in P19C16 embryonic carcinoma cells. J Muscle Res Cell Motil 2003; 24: 211-8.

14. Nakamura T, Sano M, Songyang Z, Schneider MD. A Wnt- and beta-catenin-dependent pathway for mammalian cardiac myogenesis. Proc Natl Acad Sci U S A 2003; 100: 5834-9.

15. Kioussi C, Briata P, Baek SH, et al. Identification of a Wnt/Dvl/beta-Catenin --> Pitx2 pathway mediating cell-type-specific proliferation during development. Cell 2002; 111: 673-85.

16. Briata P, Ilengo C, Corte G, et al. The Wnt/beta-catenin-->Pitx2 pathway controls the turnover of Pitx2 and other unstable mRNAs. Mol Cell 2003; 12: 1201-11.

17. Schweickert A, Campione1 M, Steinbeisser H, Blum M. Pitx2 isoforms: involvement of Pitx2c but not Pitx2a or Pitx2b in vertebrate left-right asymmetry. Mech Dev 2000; 90: 41-51.

18. Franco D, Campione M. The role of Pitx2 during cardiac development. Linking left-right signaling and congenital heart diseases. Trends Cardiovasc Med 2003; 13: 157-63. (Review)

19. Kitamura K, Miura H, Miyagawa-Tomita S, et al. Mouse Pitx2 deficiency leads to anomalies of the ventral body wall, heart, extra- and periocular mesoderm and right pulmonary isomerism. Development 1999; 126: 5749-58.

20. Maciolek NL, Alward WL, Murray JC, Semina EV, McNally MT. Analysis of RNA splicing defects in PITX2 mutants supports a gene dosage model of Axenfeld-Rieger syndrome. BMC Med Genet 2006; 7 : 59.

21. Footz T, Idrees F, Acharya M, Kozlowski K, Walter MA. Analysis of mutations of the PITX2 transcription factor found in patiens with Axenfeld-Rieger Syndrome. Invest Ophthalmol Vis Sci 2009; 50: 2599-606.

22. Martínez-Fernandez S, Hernández-Torres F, Franco D, Lyons GE, Navarro F, Aránega AE. Pitx2c overexpression promotes cell proliferation and arrests differentiation in myoblasts. Dev Dyn 2006; 235: 2930-9. 
23. Rajangam K, Behanna HA, Hui MJ, et al. Heparin binding nanostructures to promote growth of blood vessels. Nano Lett 2006; 6: 2086-90.

24. Takahashi T, Lord B, Schulze PC, et al. Ascorbic acid enhances differentiation of embryonic stem cells into cardiac myocytes. Circulation 2003; 107: 1912-6.

25. Sauer H, Rahimi G, Hescheler J, Wartenberg M. Role of reactive oxygen species and phosphatidylinositol 3-kinase in cardiomyocyte differentiation of embryonic stem cells. FEBS Lett 2000; 476: 218-23.

26. Yuasa S, Itabashi Y, Koshimizu U, et al. Transient inhibition of BMP signaling by Noggin induces cardiomyocyte differentiation of mouse embryonic stem cells. Nat Biotechnol 2005; 23: 607-11.

27. Heng BC, Haider HKh, Sim EK, Cao T, Ng SC. Strategies for directing the differentiation of stem cells into the cardiomyogenic lineage in vitro. Cardiovasc Res 2004; 62: 34-42. (Review)

28. Mommersteeg MT, Brown NA, Prall OW, et al. Pitx2c and Nkx2-5 are required for the formation and identity of the pulmonary myocardium. Circ Res 2007; 101: 902-9.

29. Molkentin JD, Antos C, Mercer B, Taigen T, Miano JM, Olson EN. Direct activation of a GATA6 cardiac enhancer by Nkx2.5: evidence for a reinforcing regulatory network of Nkx2.5 and GATA transcription factors in the developing heart. Dev Biol 2000; 217: 301-9.

30. Jamali M, Rogerson PJ, Wilton S, Skerjanc IS. Nkx2-5 activity is essential for cardiomyogenesis. J Biol Chem 2001; 276: 42252-8.

31. Watt AJ, Battle MA, Li J, Duncan SA. GATA4 is essential for formation of the proepicardium and regulates cardiogenesis. Proc Natl Acad Sci U S A 2004; 101: 12573-8.

32. Jiang Y, Drysdale TA, Evans T. A role for GATA-4/5/6 in the regulation of Nkx2.5 expression with implications for patterning of the precardiac field. Dev Biol 1999; 216: 57-71.

33. Brewer AC, Alexandrovich A, Mjaatvedt CH, Shah AM, Patient RK, Pizzey JA. GATA factors lie upstream of Nkx 2.5 in the transcriptional regulatory cascade that effects cardiogenesis. Stem Cells Dev 2005; 14: 425-39.

34. Tomita S, Li RK, Weisel RD, et al. Autologous transplantation of bone marrow cells improves damaged heart function. Circulation 1999; 100: II247-56.

35. Sakai T, Li RK, Weisel RD, et al. Autologous heart cell transplantation improves cardiac function after myocardial injury. Ann Thorac Surg 1999; 68: 2074-80.

36. Gnecchi M, He H, Liang OD, et al. Paracrine action accounts for marked protection of ischemic heart by Akt-modified mesenchymal stem cells. Nat Med 2005; 11: 367-8.

37. Gnecchi M, He H, Noiseux N, et al. Evidence supporting paracrine hypothesis for Akt-modified mesenchymal stem cell-mediated cardiac protection and functional improvement. FASEB J 2006; 20: $661-9$.

38. Noiseux N, Gnecchi M, Lopez-Ilasaca M, et al. Mesenchymal stem cells overexpressing Akt dramatically repair infarcted myocardium and improve cardiac function despite infrequent cellular fusion or differentiation. Mol Ther 2006; 14: 840-50. 